\title{
Physiological and biochemical aspects of the fungicidal action of promising biocontrol Bacillus subtilis strains against phytopathogenic fungi pp. Fusarium and Pyrenophora
}

\author{
Marina Pavlova ${ }^{1 *}$, Anzhela Asaturova, Valeria Allakhverdian, and Tatyana Sidorova \\ All-Russian Research Institute of Biological Plant Protection, Russia, Krasnodar-39, 350039
}

\begin{abstract}
The article presents some aspects of the interaction between biocontrol Bacillus subtilis strains and phytopathogenic fungi Fusarium and Pyrenophora. The presence of antifungal metabolites complexes in the culture fluid of the strains, including surfactin and iturin A has been found. The nature of the changes in the mycelium of phytopathogenic fungi is examined when co-cultivated with B.subtilis strains.
\end{abstract}

\section{Introduction}

Representatives of the genus Bacillus, and B. subtilis in particular, are common representatives of the associative microbiota of agricultural plants, and exist due to the resources provided by the plant $[1,2,3]$. During their life in the rhizosphere, these bacteria, in turn, have a positive effect on the growth and development of the host plant, including contributing to its effective protection against phytopathogens. Therefore, promising $B$. subtilis strains are often used as a basis for the development of environmentally friendly biopesticides.

The action mechanism of such biological products is based on the fact that the introduced live bacterial cells are settled and firmly fixed on the roots of the treated plant. At the same time, bacteria coexist with the plant with the mutual benefit, consuming its root secretions, and protecting the occupied ecological niche from competitors, including phytopathogenic fungi. To reach this goal, they use a number of mechanisms: from the production of antibiotics and lytic exoenzymes (proteases, chitinases, $\beta$-1,3-glucanases, etc.) $[4,5]$ to the competitive exclusion of phytopathogens $[6,7]$. The high protective effect of biological preparations based on the Bacillus bacteria is ensured by their ability to produce antibiotic substances of various classes. For example, the lipopeptides of the Bacillus bacteria play an important role in protecting plants against diseases of the fungal, bacterial

${ }^{*}$ Corresponding author: FridaAmely@yandex.ru 
and viral etiology, as well as in the development of induced systemic resistance in plants $[8,9]$.

Most scientists associate the biocidal effect of lipopeptides with their ability to disrupt the permeability of the cytoplasmic membrane, form pores in it and destroy it [10]. A special role in antibiotic activity belongs to the cyclic lipopeptides of the surfactin, iturin, and fengicin families. The action mechanism of iturins on the phytopathogenic fungus comprises the formation of ion channels that are embedded in the cytoplasmic membrane of cells, resulting in a leak of cytoplasm. Surfactins exhibit hemolytic, antiviral and antibacterial properties. Although their antifungal properties are limited, there is evidence of their effect on hyphae and spores of Fusarium moniliforme (Sacc.) Nirenberg [11], they also contribute to the protection of plants against fungal infections due to the synergistic effect on iturin and fengicin, as well as a beneficial effect on the formation of biofilms [12]. The lipopeptides of the fengicin family, which have weak hemolytic properties, unlike surfactins and iturins, have strong fungicidal activity.

The production of lipopeptides of the surfactin, iturin and fengicin families by bacterial strains is a reliable basis for the development of effective microbiological preparations based on them. Currently, these and other interaction mechanisms between rhizobacteria and plants and phytopathogens are being intensively studied, which may be one of the most promising approaches to the development of biological products for green and organic farming. That is why the aim of our research was to study the physiological and biochemical aspects of the antifungal action of promising $B$. subtilis strains against phytopathogenic fungi Fusarium, Pyrenophora, occupying various ecological niches and possessing different biology.

\section{Materials and methods}

As the research objects we used PGPR strains of bacteria $B$. subtilis BZR $336 \mathrm{~g}$ and $B$. subtilis BZR 517 and the isolates of phytopathogenic fungi Pyrenophora tritici-repentis (Died.) Drechsler BZR 7, Fusarium graminearum Schwabe BZR 4 and Fusarium oxysporum var. orthoceras App. et Wr. BZR 6 from the Bioresource collection FSBSI ARRIBPP "State Collection of Entomoacariphages and Microorganisms". The material and technical base of this study was the large-scale research facilities "Technological line for obtaining microbiological plant protection products of a new generation " (http://ckprf.ru/usu/671367/).

The antagonistic activity of the strains and microscopic changes in the mycelium of phytopathogenic fungi were studied using the modified double culture method. To carry out the research, Petri dishes with glass slides inside were poured with an agarized nutrient medium and half-seeded with a culture of $B$. subtilis strain, and a pathogen mycelium block was placed opposite. As cultures grew, the slides were removed and examined under a microscope every two days for 14 days (AxioScopeA, CarlZeiss, Germany).

The enzymatic activity of the producer-strains of the biological preparations was determined using various qualitative tests: lipase activity test with yolk agar, casein hydrolysis test, chitinase activity test [13].

The ability of bacterial strains to produce antifungal metabolites was assessed using thin layer chromatography (TLC) and bioautography [14]. TLC of the ethyl acetate extract of the culture fluid (CL) of the studied strains was carried out on KieselgelMerck chromatographic plates, with the layer thickness $2 \mathrm{~mm}$, in a solvent system ethyl acetate ethanol - water 40:15:15. Bioautography was carried out using a potato-glucose medium; the fungus $F$. oxysporum var. orthoceras was used as a test object. The presence of antifungal metabolites, their chromatographic mobility and degree of activity were judged by the size and position of the zones of growth inhibition of the fungus. 


\section{Results and discussion}

As a result of step-by-step screening according to the criteria of antifungal and growthstimulating activity, biological efficacy against an artificial infectious background, and others, we selected two most promising strains among more than 1000 strains of BRC of FSBSI ARRIBPP: B. subtilis BZR 336g and B. subtilis BZR 517. Based on these strains, experimental samples of biological products are being studied and developed to protect winter wheat and other crops against economically significant diseases. During many years of laboratory and field studies, they showed relatively high biological efficacy against Fusarium root rot and spike Fusarium: $23-71 \%$ and 16-73 \%, respectively, as well as against yellow leaf spot - 38-94\%. Currently, an experimental sample based on the $B$. subtilis BZR $336 \mathrm{~g}$ strain is in the registration test stage.

The biological efficacy of the strains against these diseases is based on their ability to suppress the development of phytopathogenic fungi, causing pathological changes in the structure and functioning of fungal hyphae. Microscopic studies of $F$. graminearum and $P$. tritici-repentis mycelium, when co-cultured with the studied strains, revealed a shortening of the hyphal segments by 2.5-4.0 times and their thickening by 2.0-2.5 times.

Moreover, when co-cultured with $B$. subtilis strains, some mycelium segments of $F$. graminearum assumed an almost spherical shape («chlamydospore» type cells). Often this was accompanied by a rupture of the hyphae with the outside release of the contents. Degenerative changes in intracellular membrane structures were also observed ("vacuolization"), resembling damage under osmotic stress (Fig. 1a)
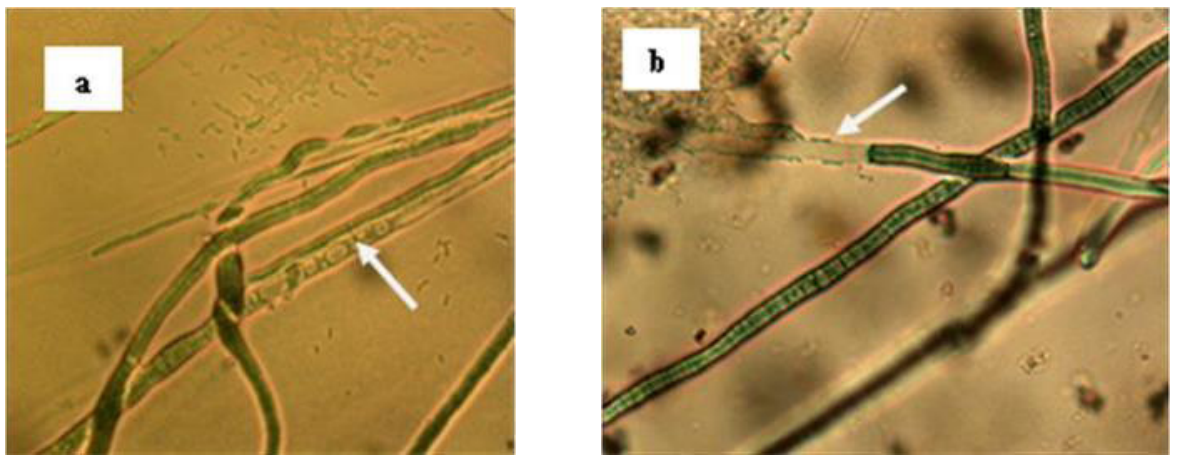

Fig. 1. F. graminearum mycelium when co-cultured with $B$. subtilis BZR 517 strain - vacuolization of the contents of hypha (a), cells of B. subtilis BZR 336g strain on the surface of hypha (b), $400 \times$ magnification

Since the 7-10th day of co-cultivation of F. graminearum with B. subtilis strain BZR $336 \mathrm{~g}$, bacterial chemotaxis in the direction of hyphae, the movement of bacterial cells along the surface of hyphae (Fig. 1b), and their gradual lysis were observed.

In the double cultures of $B$. subtilis strains with $P$. tritici-repentis, bacterial cells did not come into contact with fungal hyphae due to the fact that mycelium growth stopped at a considerable distance from the bacterial culture. The pathogen hyphae were thickened, deformed, and intensively branched, however, the formation of spherical cells or the vacuolization of contents, similar to that observed in F. graminearum, was recorded only in some single cases. On the 5-7th day of co-cultivation of $P$. tritici-repentis with biocontrol B. subtilis strains, pink pigmentation of the mycelial edge became noticeable. Microscopy 
revealed that the pigment is localized on the surface of hyphae in the form of needle structures (Fig. 2).

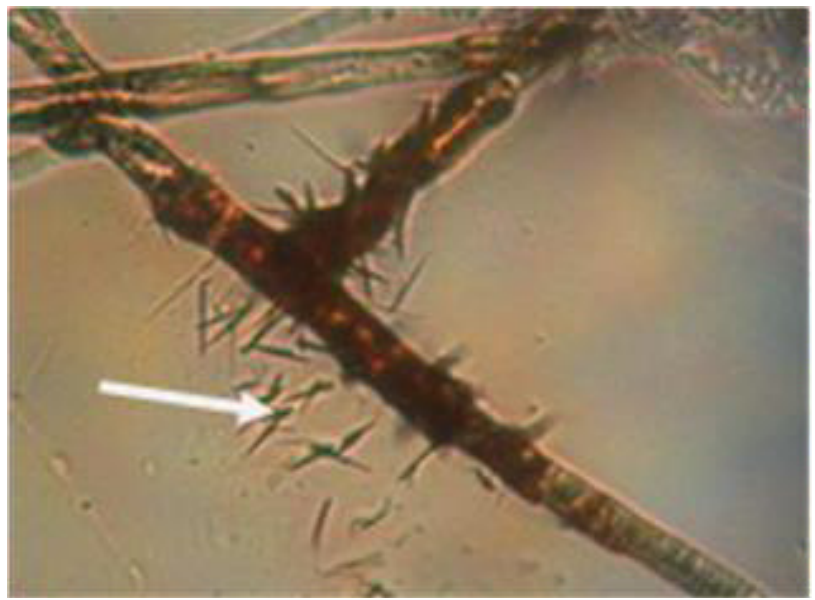

Fig. 2. P. tritici-repentis mycelium when co-cultured with B. subtilis BZR 336g: needle structures on the surface of pigmented hyphae; magnification $400 \times$

Presumably, the observed pigmentation may be due to the release of cynodontin (1,4,5,8-tetrahydroxy-2-methylanthraquinone), which is found in Drechslera avenae under the influence of biotic and abiotic stress [15]. Thus, using microscopic methods we found that the representatives of two groups of phytopathogenic fungi with fundamentally different biology, exhibit both similar and different reactions to the effects of biocontrol $B$. subtilis strains.

Qualitative tests for enzymatic activity showed that the studied B. subtilis BZR $336 \mathrm{~g}$ and $B$. subtilis BZR 517 strains exhibit high caseinolytic activity, and B. subtilis BZR 336g strain also exhibits lipolytic activity. None of the strains exhibitted chitinolytic activity.

The study of antifungal metabolites of B. subtilis BZR $336 \mathrm{~g}$ and B. subtilis BZR 517 strains showed the following. The bioautograms of the ethyl acetate extracts of the both strains LC revealed zones of growth inhibition of the test fungus, which corresponds to the chromatographic profile of bacterial antifungal metabolites. Moreover, the degree of fungitoxicity of the compound can be judged by the nature of the suppression, since zones of both complete suppression (fungicidality) and partial (fungistaticity) are detected. Several such zones were found in the bioautograms of both B.subtilis strains, which indicates a high fungicidal potential of the bacteria (Fig. 3).

The use of lipopeptide standards in the experiment, such as surfactin, iturin A, and fengicin, made it possible to draw a conclusion about their presence in the studied culture liquids. Moreover, both the chromatographic mobility (Rf) of the substance and the nature of the fungus growth in the suppression zone were taken into account. Thus, a significant inhibition zone of the test fungus with Rf 0.78 was found in the CL bio-autogram of $B$. subtilis BZR 336g strain, which corresponds to Rf of surfactin, a standard under the given conditions of TLC (Fig. 3). The growth pattern of the fungus in the analyzed zone is also visually identical to that in the surfactin zone. A similar zone of the fungal growth inhibition was also found in the bioautogram of the CL of B. subtilis BZR 517 strain, but a visual assessment of the degree of the fungus inhibition suggests that it is lower than that of the CL of B. subtilis BZR 336g. 


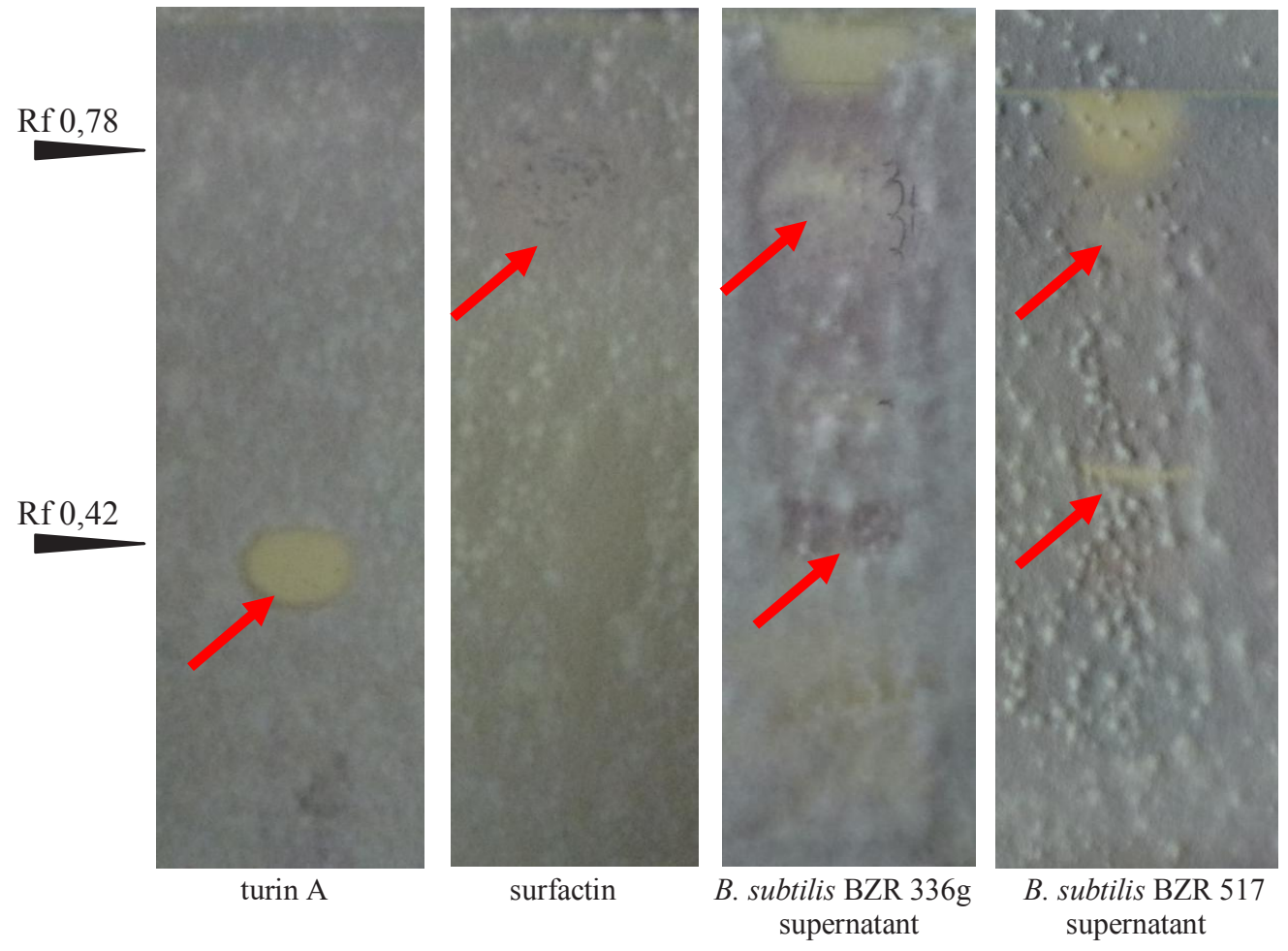

Fig.3. Comparison of isolated B. subtilis BZR 336g (3) and B. subtilis BZR 517 (4) metabolites with standard antifungal metabolites

When considering the zone of the fungal growth inhibition with $\mathrm{Rf} 0.42$, which corresponds to the chromatographic mobility of iturin A - standard (Fig. 3), it should be noted that in this zone the fungus practically stops growing - complete fungicidal activity. This can indicate either a high activity of the compound or its high concentration in the CL. Zones of the fungal growth inhibition that are close in Rf to surfactin and iturin A are likely to be formed by compounds of the families of these lipopeptides. As for fengicin, it was not found on the CL bioautograms of both studied strains, although its presence in small quantities cannot be ruled out.

Thus, B. subtilis BZR 336g and B. subtilis BZR 517 bacterial strains are capable of synthesizing the antifungal lipopeptides surfactin and iturin A during metabolism, and thereby have a detrimental effect on test cultures of the phytopathogenic fungus.

It's nteresting that the CLs of B. subtilis BZR 336g and B. subtilis BZR 517 strains grown even in the absence of phytopathogenic fungi contain substances that inhibit their development. Antifungal metabolites of the studied strains, apparently, are not highly specific against certain pathogens, but have a wide spectrum of action, inhibiting the development of fungi of various systematic groups. Most of the mycelium damage due to antifungal metabolites is believed to be due to osmotic cell stress. In the case of close contact of the strains with phytopathogenic fungi, bacteria actively move in the direction of fungal hyphae and can completely destroy them and use them as a food source.

Therefore, we've come to the conclusion that the studied B. subtilis strains have a wide spectrum of antifungal activity, apparently of a nonspecific nature, due to the synthesis of ectoenzymes and antifungal antibiotics, including surfactin and iturin A. All these facts allow us to judge the high ecological plasticity of B. subtilis BZR $336 \mathrm{~g}$ and B. subtilis BZR 
517 strains and their ability to control the development of phytopathogenic fungi not only in the rhizosphere, but in other ecological niches of plants.

The studies were performed in accordance with State Assignment No. 075-00376-19-00 of the Ministry of Science and Higher Education of the Russian Federation as part of research on topic № 0686-2019-0013.

\section{References}

1. R.R. Azizbekyan, Biotech., 35, 69 (2013) https://doi.org/10.21519/0234-2758-2019$\underline{35-3-3-11}$

2. K. Ntushelo, L.K. Ledwaba, M.E. Rauwane, O.A. Adebo, P.B. Njobeh, Toxins, 11, 606 (2019) https://doi.org/10.3390/toxins12040216

3. J. Ma, C. Wang, H. Wang, K. Liu., T. Zhang, L. Yao, Z. Zhao., B. Du, Y. Ding, Biomed. Res. Int. 6, 9473542 (2018) http://doi.org /10.1155/2018/9473542

4. T. Yu. Gagkaeva, O.P. Gavrilova, A.I Kuzin., N.I Kuznetsova., M.A Nikolaenko, Biotechn. in Russ. 30, 32 (2014) https://elibrary.ru/item.asp?id=24083400

5. I. V. Maksimov, R.R Abizgil'dina, L.I Pusenkova, App. Biochem. and Microbiol., 47, 333 (2011) https://doi.org/10.1134/s0003683811040090

6. S. Caulir, C. Nannan., A. Gillis, F. Licciardi, C. Bragard, J. Mahillon, Front Microbiol., (2019) https://doi.org/10.3389/fmicb.2019.00302

7. R. O. Penha, L. P. S. Vandenberrghe, C. Fauls, V. T. Soccol, Microb. Biotechnol., 8, 281 (2015) https://doi.org/10.1007/s00425-020-03357-7

8. H. Cawoy, D. Debois, L. Franzil, E. De Pauw, P. Thonart, M. Ongena, Microb.

Biotechnol. 8, 281 (2015) https://doi.org/10.1111/1751-7915.12238

9. G. Gupta, S.S Parihar, N.K Ahirwar, S. K. Snehi, V. Singh, J. Microb. Biochem. Technol., 7, 096 (2015) https://doi.org/10.4172/1948-5948.1000188

10. S. Fiedler, H. Heerklotz, Biophys. J., 109, 2079 https://doi.org/10.1016/j.bpj.2015.09.021

11. J. Jiang., L. Gao, X. Bie, Z. Lu, H. Liu, C. Zhang, F. Lu, H. Zhao, BM

Microbiology, 31 (2016) https://doi.org/10.1186/s12866-016-0645-3

12. L.S. Cairns, L. Hobley, N.R. Stanley-Wall, Mol. Microbiol., 93, 587 (2014)_ https://doi.org/10.1111/mmi.12697

13. L. G Ortiz, I. Z. Quintero, Afr. J. of Botech., 15, 408 (2016). https://doi.org/10.5897/ajb2015.14991

14. T.M. Sidorova, A.M.Asaturova, A.I. Homyak, N.S Tomashevich, Agricult. biol., 54, 178 (2019) https://doi.org/10.15389/agrobiology.2019.1.178eng

15. N.N. Gessler, A.S Egorova, T.A. Belozerskaya, Appl. Biochem. and Microb, 49, 85 (2013) https://doi.org/10.1134/s000368381302004x 